\title{
Toroidal embeddings of abstractly planar graphs are knotted or linked
}

\author{
Senja Barthel ${ }^{1}$ • Dorothy Buck ${ }^{1}$
}

Received: 22 January 2015 / Accepted: 19 May 2015 / Published online: 31 May 2015

(C) The Author(s) 2015. This article is published with open access at Springerlink.com

\begin{abstract}
We give explicit deformations of embeddings of abstractly planar graphs that lie on the standard torus $T^{2} \subset \mathbb{R}^{3}$ and that contain neither a nontrivial knot nor a nonsplit link into the plane. It follows that ravels do not embed on the torus. Our results provide general insight into properties of molecules that are synthesized on a torus.
\end{abstract}

Keywords Topological graphs · Templating on a toroidal substrate .

Knots and links

Mathematics Subject Classification $\quad 05 \mathrm{C} 10 \cdot 92 \mathrm{E} 10 \cdot 57 \mathrm{M} 25$

\section{Introduction}

The interaction between mathematical topology, in particular topological graph theory, and the investigation of chemical structures is a rich area ([1-7] and the references therein). Topological graph theory studies embeddings of graphs in 3-space. Those spatial graphs can be thought of as knots or links with additional edges attached: a spatial graph is the image $\mathcal{G}$ of an embedding $f: G \rightarrow \mathbb{R}^{3}$, where $G$ is an abstract graph. Two spatial graphs are considered different if it is not possible to transform one into the other without self-intersections during the transformation (note that an abstract graph has many different spatial graph realisations). Ambient isotopies such

\footnotetext{
$\triangle$ Senja Barthel

s.barthe111@imperial.ac.uk

Dorothy Buck

d.buck@imperial.ac.uk

1 Imperial College London, London, UK
} 
as bending, stretching and shrinking of edges are allowed as long as no edge is shrunk to a point.

Spatial graphs can model molecules. For example, embeddings of bonding graphs correspond to spatial graphs. Also, the ligands of a coordination polymer correspond to edges of a spatial graph and the coordination entities correspond to its vertices. Results about spatial graphs directly translate to information about the configuration of molecules. In particular, if entangled chemical structures like knots, links, braids and ravels are present, topological graph theory can be an appropriate framework [1-7].

As molecules with non-standard topological structure often have unusual chemical properties, synthetic organic chemists have designed new structures that include entanglements (e.g. [8-19]). Furthermore, crystal engineers have produced coordination networks that contain knots and links [6]. Many 3-dimensional and several 2-dimensional entangled structures have been reported by experimentalists [20,21]. A concept of topological entanglements called ravels that are not caused by knots or links was introduced to chemistry by Hyde et al. [22]. Following, a mathematical description in terms of spatial graphs of one family of ravels was given by Flapan et. al. [23]. A molecular ravel was synthesised by Lindoy et al. [9].

Knot theoretical methods can predict, or give constraints on, the possible entanglements and related properties like chirality of chemical structures.

Many molecules have a corresponding abstractly planar graph, i.e. an abstract graph for which an embedding on the sphere $S^{2}$ (equivalently on the plane $\mathbb{R}^{2}$ ) exists. Such an embedding is a planar embedding and its image is a planar spatial graph. Embeddings of molecules on a sphere (respectively planar spatial graphs) contain no nontrivial knots or nonsplit links. A ravel is a nonplanarly embedded $\theta_{n}$-graph that does not contain a nontrivial knot. The $\theta_{n}$-graph consists of two vertices that are joined by $n$ edges; it cannot contain links.

We are interested in molecules with a corresponding abstractly planar graph which are flexible enough to be realised in different forms in 3-space, in particular as knotted molecules. Examples of such flexible molecules are carcerands or molecules that are built with DNA strands. The next more topologically complex surface in $\mathbb{R}^{3}$ after the sphere is the torus, and embeddings on the standard torus can be nontrivially knotted and linked. Whenever we use the word torus in the rest of this paper, we refer to the standard torus. Therefore it is reasonable to investigate how molecules with abstractly planar underlying graphs can embed on the torus. We call graph embeddings (and molecules that are described by them) on the torus that do not embed on the sphere toroidal.

Following Hyde, a polyhedral molecule has an underlying graph that is abstractly planar 3-connected and simple. (A graph is $n$-connected if at least $n$ vertices and their incident edges have to be removed to disconnect the graph or to reduce it to a single vertex. A graph is simple if it has neither multiple edges between a given pair of vertices nor loops from a vertex to itself.)

Conjecture 1 (Castle et al. [24]) All polyhedral toroidal molecules contain a nontrivial knot or a nonsplit link. 

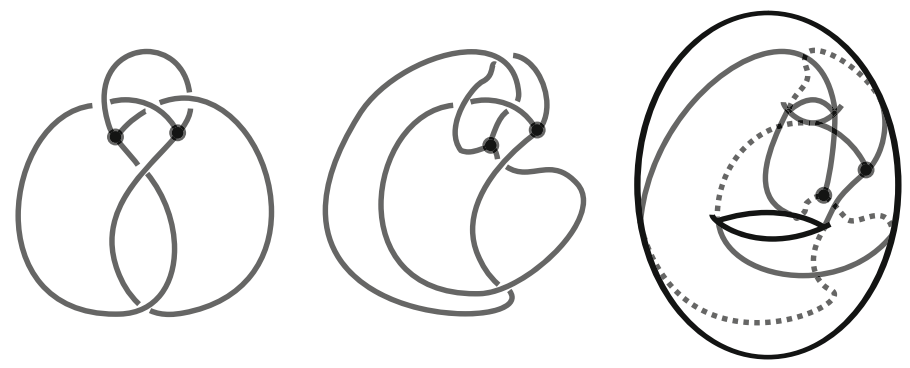

Fig. 1 The Kinoshita-theta curve can be embedded on the genus 2 surface

The main result (Theorem 1) proves this conjecture without assuming 3-connectivity or simpleness using topological graph theory. The argument gives an explicit deformation from embeddings of abstractly planar graphs on the torus that contain neither a nontrivial knot nor a nonsplit link into the plane. A much shorter but less intuitive proof that relies on deep theorems of topological graph theory is given in [25]. The argument on hand not only presents a self-sufficient argument but will hopefully also give the reader a better feeling for the nature of graphs that are embedded on the torus.

Theorem 1 (Existence of knots and links) Let $G$ be an abstractly planar graph and $f: G \rightarrow \mathbb{R}^{3}$ be an embedding of $G$ with image $\mathcal{G}$. If $\mathcal{G}$ lies in the torus $T^{2}$ and contains no subgraph that is a nontrivial knot or a nonsplit link, then the embedding $f$ is planar.

Corollary 1 (Ravels do not embed on the torus) Every nontrivial embedding of a $\theta_{n}$-graph on the torus contains a nontrivial knot.

The topological structure of the surface on which the spatial graph is embedded is crucial for the theorem. For all closed orientable surfaces of genus $g>1$, there exist examples of abstractly planar spatial graphs that are neither knotted or linked nor embeddable on a closed orientable surface with genus less than $g$. A famous example for the closed oriented genus two surface is Kinoshita's theta curve [26] (Fig. 1). A big class of examples is given by ravels: a ravel corresponds to an abstractly planar spatial graph and does not embed on the torus by Theorem 1 as a ravel is nonplanar but contains no nontrivial knot or nonsplit links by definition. As every spatial graph embeds on a compact closed surface of some genus, it follows that ravels correspond to spatial graphs that are neither planar embedded nor knotted or linked but which are realisations of abstractly planar graphs on higher genus surfaces. Again, one needs arbitrarily high genera to accommodate all ravels which can be shown using a Borromean construction as given by Suzuki [27].

Castle et al. [24] proved that polyhedral toroidal molecules which contain a nontrivial knot are chiral. The chirality of polyhedral toroidal molecules which contain a nonsplit link is shown in [28]. Note that topological chirality implies chemical chirality.

Theorem 2 (Chirality [24,28]) Let $G$ be a simple 3-connected abstractly planar graph and $f: G \rightarrow T^{2} \subset \mathbb{R}^{3}$ be an embedding of $G$ with image $\mathcal{G}$ on the torus $T^{2}$. If $\mathcal{G} \subset T^{2}$ is nonplanar embedded, then $\mathcal{G}$ is topologically chiral in $R^{3}$. 


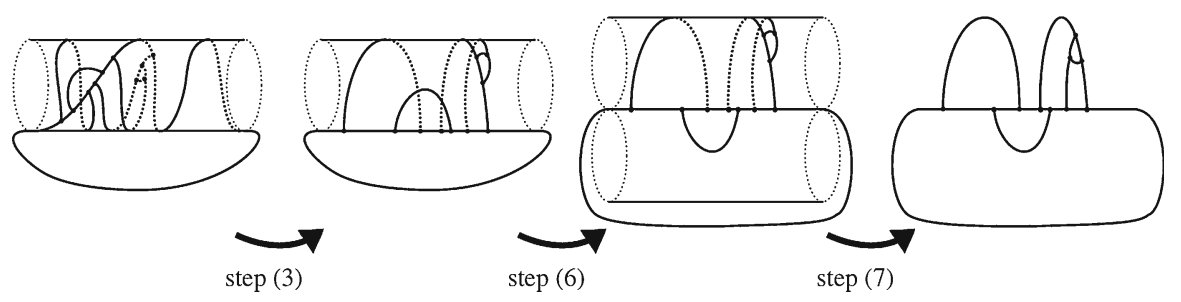

Fig. 2 The ambient isotopy of the proof in Sect. 2.2

\section{Proof of the theorem by giving an explicit isotopy}

\subsection{Outline of the proof of Theorem 1 and preparations}

The idea of the proof of Theorem 1 is the following: let $G$ be an abstractly planar graph and $\mathcal{G}$ be the spatial graph that is the image of the embedding $f: G \rightarrow T^{2}$. Assume that $\mathcal{G}$ contains neither a nontrivial knot nor a nonsplit link. Find a general construction for an explicit ambient isotopy in $\mathbb{R}^{3}$ from the spatial graph $\mathcal{G}$ to a spatial graph $\mathcal{G}^{\prime}$ which is embedded in the plane $\mathbb{R}^{2} \subset \mathbb{R}^{3}$. This demonstrates that any embedding of an abstractly planar graph that is embedded on the torus and contains neither a nontrivial knot nor a nonsplit link is planar. The ambient isotopy is illustrated in Fig. 2.

To construct the ambient isotopy in $\mathbb{R}^{3}$ from the embedding $\mathcal{G} \subset T^{2}$ of an abstractly planar graph $G$ that contains neither a nontrivial knot nor a nonsplit link to a spatial graph $\mathcal{G}^{\prime}$ which lies in the plane $\mathbb{R}^{2} \subset \mathbb{R}^{3}$, we first note that it is sufficient to consider connected graphs by Lemma 1. Furthermore, we will see in step (1) of the proof that it is sufficient to restrict to spatial graphs that have a subgraph of the form $T(1, n), n>0$ as otherwise, the graphs would be abstractly nonplanar or would be already planar embedded. Then, the desired ambient isotopy of the graph consists of three deformations. The first one is a twist around the core of the torus which transforms $T(1, n)$ into the longitude $l$. This twist is described in step (3). Step (2) of the proof is a technicality that ensures the existence of the twist given in step (3) by arguing that there exists a meridian of $T^{2}$ that intersects $\mathcal{G}$ in only one point. As $G$ is abstractly planar by assumption, it follows from Theorem 3 that the conflict graph of $G$ with respect to $l$ is bipartite. The bipartiteness of the conflict graph is used together with the property of the spatial graph being embedded on the torus to show in step (4) and step (5) of the proof that a second ambient isotopy can be performed. This ambient isotopy is given in step (6). It rotates the spatial fragments in space around the longitude $l$, so that $\mathcal{G}$ is ambient isotoped to a spatial graph that is embedded on the surface $8 \times S^{1}$, so that conflicting spatial fragments lie in different components of $\left(8 \times S^{1}\right) \backslash\left(P \times S^{1}\right)$. We use 8 to denote a loop with one point $P$ of self-intersection. Step (7) shows that each spatial fragment can individually ambient isotoped to a planar embedded spatial fragment in space, independently from all other spatial fragments. The combination of the ambient isotopies given in step (3), step (6) and step (7) gives the desired deformation of $\mathcal{G}$ into the plane which proves Theorem 1 .

To work on the torus, define the following: a meridian of a solid torus $T$ is an essential simple closed curve in $\partial T$ that bounds a disc in $T$. (An essential simple 
Fig. 3 The abstract graph on the left side has a bipartite conflict graph which is shown on the right

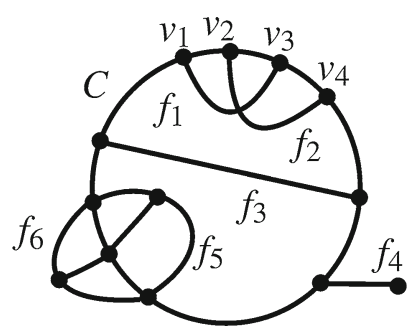

closed curve in $\partial T$ does not bound a disc in $\partial T$.) The preferred longitude is a simple closed curve in $\partial T$ that intersects the meridian once and has linking number zero with the core of the torus $T$. For the standard torus $T^{2}$ in $\mathbb{R}^{3}$ define the meridian and preferred longitude analogously by taking the interior of the solid torus to be the bounded component of $\mathbb{R}^{3} \backslash T^{2}$. Whenever we use the term longitude in this paper, we will refer to the preferred longitude.

To describe knots and links on the torus, we define a torus knot or torus link to be a knot or link that is embedded on the standard torus $T^{2}$ following the longitude of the standard torus $p$ times and the meridian $q$ times. Those knots or links are denoted by $T(p, q)$ with $p, q$ integers. Therefore, a meridian is denoted by $m=T(0,1)$ and a longitude by $l=T(1,0)$. An unknot that bounds a disc in $T^{2}$ is denoted by $T(0,0)$.

The concept of the 'conflict graph' defined below will be needed (compare Fig. 3), to ensure abstract planarity of the considered graphs during the proof:

A cycle is a simple closed path in a graph or in a spatial graph. Let $C$ be a cycle in a graph $G$. The connected components $f_{i}$ of the graph $G \backslash C$ are called fragments of $G$ with respect to $C$ and two fragments $f_{i}$ and $f_{j}$ conflict if and only if at least one of the following conditions is satisfied:

- There exist three points on $C$ to which both components $f_{i}$ and $f_{j}$ are attached to.

- There exist four interlaced points $v_{1}, v_{2}, v_{3}, v_{4}$ on $C$ in cyclic order so that $f_{i}$ is attached to $C$ at $v_{1}$ and $v_{3}$ and $f_{j}$ is attached to $C$ at $v_{2}$ and $v_{4}$.

Two sets $\left\{p_{1} \ldots p_{n}\right\}$ and $\left\{q_{1} \ldots q_{m}\right\}$ of points on $C$ are nested, if all points of one set lie in between two points of the other set. The elements of two nested sets do not conflict by definition. Let $f_{i}$ be a fragment of $G$ with respect to $C$. Call the set of points in which $f_{i}$ is attached to $C$ the endpointset $v\left(f_{i}\right)$ of the fragment $f_{i}$. Note that vertices of $C$ can be elements of different endpointsets $v\left(f_{i}\right)$ and $v\left(f_{j}\right)$ where $f_{i}$ and $f_{j}$ might or might not conflict. Two fragments $f_{a}$ and $f_{b}$ are nested if their endpointsets on $C$ are nested. If a basepoint on $C$ is given and a pair of nested fragments, call the fragment outer fragment whose endpoints are first reached form the basepoint. Its endpointset is called outer points. Call the other fragment inner fragment and its endpointset inner points. The restriction of a spatial graph to a fragment is a spatial fragment.

The conflict graph of a cycle $C$ in a graph $G$ is constructed by introducing a vertex $u_{i}$ for every fragment $f_{i}$ of $G$ with respect to $C$ and adding an edge between the vertices $u_{i}$ and $u_{j}$ if and only if the fragments $f_{i}$ and $f_{j}$ conflict. Fix a point $p$ on $C$, take an orientation of $C$ and a parametrisation $f:[0,2 \pi] \rightarrow C, f(0)=f(2 \pi)=p$ that respects the orientation. Then for two points $a$ and $b$ on $C$, we say that $a<b$ if $f^{-1}(a)<f^{-1}(b)$. 
During the following proof, we will use the abstract planarity of $G$ in form of the following statement:

Theorem 3 (Tutte's Theorem [29]) A graph $G$ is abstractly planar if and only if the conflict graph of every cycle in $G$ is bipartite.

A graph is bipartite if its vertices can be divided into two disjoint sets $S_{1}$ and $S_{2}$ such that every edge of the graph has one endpoint in $S_{1}$ and the other endpoint in $S_{2}$.

Remark 1 (Cases of non-conflicting fragments) Let $f_{a}$ and $f_{b}$ be two fragments of a connected graph with respect to a cycle $C$. Let $v\left(f_{a}\right)=v_{a 1}, \ldots, v_{a r}$ be the endpointset of $f_{a}$ and let $v\left(f_{b}\right)=v_{b 1}, \ldots, v_{b r}$ be the endpointset of $f_{b}$. Then it follows from the definition of conflicting that $f_{a}$ and $f_{b}$ do not conflict if and only if $f_{a}$ or $f_{b}$ are attached to $C$ in one point only or if for all elements $v\left(f_{a}\right)$ and $v\left(f_{b}\right)$ (up to transposition of $a$ and $b$ ) one of the following two cases holds:

1. $v_{a 1}<\cdots<v_{a r} \leq v_{b 1}<\cdots<v_{b r}$

2. there exist two points $v_{a i}$ and $v_{a(i+1)}$ in $v\left(f_{a}\right)$ so that $v_{a i} \leq v_{b 1}, \ldots, v_{b r} \leq v_{a(i+1)}$

The sets $v_{a 1}, \ldots, v_{a r}$ and $v_{b 1}, \ldots, v_{b r}$ in the second case above are nested; the inner points are the points $v_{b 1}, \ldots, v_{b r}$.

Lemma 1 (Connectivity Lemma [25]) The image $\mathcal{G}$ of an embedding $f: G \rightarrow T^{2} \subset$ $\mathbb{R}^{3}$ of a graph $G$ with $n>1$ connected components on the torus $T^{2}$ contains either a nonsplit link, or contains no nonsplit link and decomposes into $n$ disjoint components of which at least $n-1$ components are planar embedded in $R^{3}$.

\subsection{Proof}

\section{Proof (Theorem 1)}

1. Reducing the types of spatial graphs We show that it is sufficient to consider connected abstractly planar spatial graphs that are embedded on the torus $T$ and do neither contain a nontrivial knot nor a nonsplit link, but do contain a torus unknot $T(1, n), n>0$.

We can assume that the graph $G$ is connected by Lemma 1. Furthermore, we claim that it is sufficient to consider spatial graphs $\mathcal{G}$ that contain a torus unknot of the form $T(1, n), n>0$ [respectively $T(n, 1)$ ] since if the only knot types contained in $\mathcal{G}$ are $T(0,0), T(0,1)$ and $T(1,0)$, then $\mathcal{G}$ is planar. We see this below by a case study where we restrict the knot types that occur in the spatial graph $\mathcal{G}$. Let $\# K$ denote the number of disjoint copies of the knot $K$.

(a) $\# T(0,0)=n$

If the only knot type contained in the spatial graph $\mathcal{G}$ is $T(0,0)$, there exists a meridian and a longitude of the torus that do not intersect $\mathcal{G}$. Therefore, $\mathcal{G}$ is planar.

(b) $\# T(0,0)=n, \# T(0,1)=k$ [respectively $\# T(0,0)=n$, $\# T(1,0)=k]$

There exist either a meridian or a longitude of the torus that does not intersect $\mathcal{G}$. Therefore, $\mathcal{G}$ is planar. 

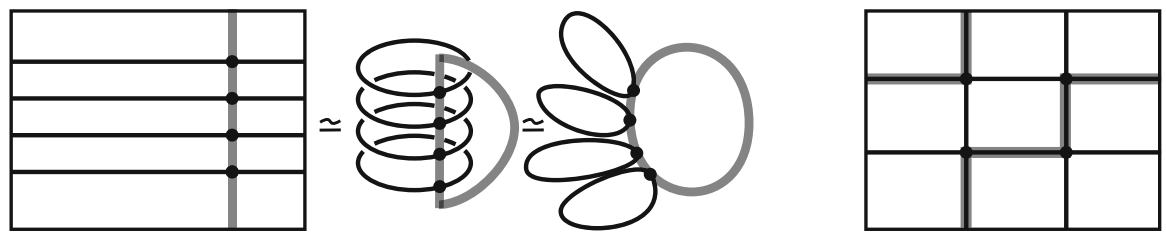

Fig. 4 Left if $\mathcal{G}$ contains only one copy of $T(0,1)$, or respectively $T(1,0)$, then $\mathcal{G}$ is planar. Right if $\mathcal{G}$ contains disjoint copy of both $T(0,1)$ and $T(1,0), \mathcal{G}$ also contains the unknot $T(1,1)$

(c) $\# T(0,0)=n, \# T(0,1)=1, \# T(1,0)=k$

$[$ respectively $\# T(0,0)=n, \# T(1,0)=1, \# T(0,1)=k]$

$\mathcal{G}$ is planar (see left of Fig. 4).

(d) $\# T(0,0)=n, \# T(0,1)=k, \# T(1,0)=m$ with $k, m>1$

This case does not fulfil the assumptions since $\mathcal{G}$ also contains the unknot $T(1,1)$ (see right of Fig. 4).

2. Existence of a meridian of $T^{2}$ that intersects $\mathcal{G}$ in only one point: Beside for some elementary cases that can be investigated directly, we show this with a Morsetheoretical argument that gives a contradiction: if every meridian of $T^{2}$ would intersect $\mathcal{G}$ in at least two points and if $\mathcal{G}$ would have a subgraph $T(1, n), n>0$, then $\mathcal{G}$ would contain either a nontrivial knot or a nonsplit link.

Note that the case where $\mathcal{G}$ is the union of $T(1, n)$ and a longitude does not fulfil the assumptions since there exists a meridian that intersect $\mathcal{G}$ in one point only (there are $n$ such meridian, namely one for each intersections of $T(1, n)$ with the longitude).

To construct a contradiction, assume that every meridian of $T^{2}$ intersects $\mathcal{G}$ in at least two points. Cut $T^{2}$ along a meridian that intersects the graph with minimal number to get a cylinder $[0,1] \times S^{1}$. Define the projection function $f:[0,1] \times S^{1} \rightarrow$ $[0,1] ;\{x, \alpha\} \mapsto x$. Let $S$ be the set of all pairs of pairwise different paths on the cylinder where one path forms a knot of type $T(1, n)$ and the other path has an endpoint in $f^{-1}(0)$ (Fig. 5a). The set $S$ is non-empty because there exists at least one cycle $T(1, n)$ by step (1) and a second path $\pi$ with endpoint in $f^{-1}(0)$ by the assumption that every meridian of $T^{2}$ intersects $\mathcal{G}$ in at least two points. Note that $\pi$ either intersects $T(1, n)$ or has the second endpoint on $f_{1}(1)$ otherwise since the number of intersections between $f^{-1}(0) \cap \mathcal{G}$ is minimal by assumption. Now take a pair of paths in $S$ which maximises the value $f\left(t_{n}\right)$ where $t_{n}$ is the intersection point of the two paths in the pair.

If $T(1, n)$ and $\pi$ do not intersect, then there exist two disjoint paths on the cylinder. There exists a nontrivial knot or a nonsplit link since the graph is connected as we show now:

If $\pi$ is a cycle, a nonsplit link is formed by $T(1, n)$ and $\pi$ (Fig. $5 b$ ). So let $\pi$ be a path with endpoints $(0, \alpha) \in f^{-1}(0)$ and $(1, \beta) \in f^{-1}(1), \alpha \neq \beta$. As the graph intersects $f^{-1}(0)$ and $f^{-1}(1)$ in a minimal number, wlog we can assume that there exist paths $\pi_{0}$ from $(0, \beta)$ and $\pi_{1}$ from $(1, \alpha)$ to $T(1, n)$ or to $\pi$ (since the graph is connected). 
[h]

(a)

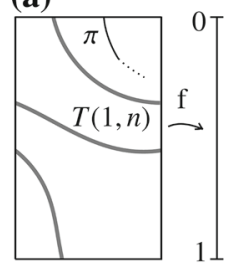

(b) $(0, \alpha)$

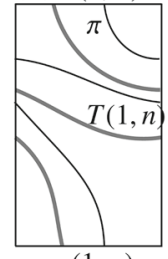

$(1, \alpha)$ (c) $(0, \alpha)$

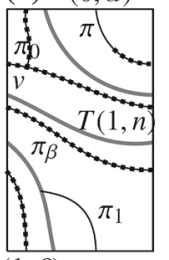

$(1, \beta)$

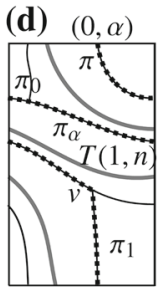

$(1, \beta)$

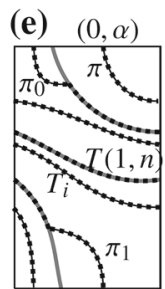

$(1, \beta)$

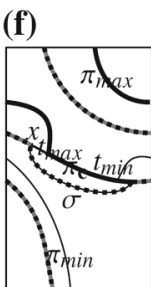

Fig. 5 Illustrating step 2 of the proof

(a) If $\pi_{0}$ intersects $\pi$ in a point $v$ before intersecting $T(1, n)$ (Fig. 5c), denote the segment of $\pi$ between $v$ and $(1, \beta)$ by $\pi_{\beta}$. Such a graph contains a nonsplit link where one component is $T(1, n)$ and the other consists of $\pi_{0}$ and $\pi_{\beta}$.

If $\pi_{1}$ intersects $\pi$ in a point $v$ before intersecting $T(1, n)$ (Fig. $5 \mathrm{~d}$ ), denote the segment of $\pi$ between $(0, \alpha)$ and $v$ by $\pi_{\alpha}$. Again, such a graph contains a nonsplit link where one component is $T(1, n)$ and the other consists of $\pi_{\alpha}$ and $\pi_{1}$. Up to this point the arguments applies to $k=T(1,1)$ as well.

(b) If both paths $\pi_{0}$ and $\pi_{1}$ intersect $T(1, n)$ before they intersect $\pi$ (Fig. 5e), denote the segment (possibly a point) of $T(1, n)$ that lies between the endpoints of $\pi_{0}$ and $p i_{1}$ by $T_{i}$. The cycle that runs through $\pi, \pi_{0}, T_{i}$ and $\pi_{1}$ is a nontrivial knot for $n>1$.

If $T(1, n)=T(1,1)$, there are possibilities to connect $(0, \beta)$ and $(1, \alpha)$ to $T(1,1)$ without introducing a nontrivial knot or a nonsplit link. But considering $T(1,1)$, we could exchange the meridian and the longitude in the argument. This gives the extra condition that not only each meridian but also each longitude intersects the graph in at least two points. An elementary investigation shows directly that Theorem 1 is valid in those cases.

If $T(1, n)$ and $\pi$ do intersect, there exists a point of maximal intersection $t_{\text {max }}, 0<f\left(t_{\text {max }}\right)<1$. Now, consider the set of all paths that are different from $T(1, n)$ and that have one endpoint on $f^{-1}(1)$. Take one path of this set which minimises the value of $f\left(t_{\min }\right)$ where $t_{\min }$ is the point of intersection between the path and $T(1, n)$ and call that path $\pi_{\text {min }}$. Denote the component of $\mathcal{G}-T(1, n)$ that contains $\pi_{\min }\left(\pi_{\max }\right)$ by $c_{\min }\left(c_{\max }\right)$.

If $f\left(t_{\min }\right)<f\left(t_{\max }\right)$, the argument is very similar to the case above.

So let us finally consider the case where $f\left(t_{\max }\right) \leq f\left(t_{\min }\right)$ (Fig. 5f). As before, since the graph intersects every meridian at least twice by assumption, there exists a point $x \not \subset \pi$ in the graph so that $f(x)=f\left(t_{\max }\right)$. No path disjoint from $T(1, n)$ containing $x$ can connect to $c_{\text {min }}$ as this would contradict the maximality of $t_{\text {max }}$. Similarly, no path disjoint from $T(1, n)$ containing $x$ can connect to $c_{\max }$ as this would contradict the minimality of $t_{\min }$. Therefore, every path through $x$ connects to $T(1, n)$ before and after $t_{\max }$ (and similarly before and after $t_{\min }$ ) and is disjoint from $c_{\max }$ and $c_{\min }$. Take such a path and denote it by $\sigma$. Replacing the segment $T_{i}$ of $T(1, n)$ that runs between the endpoints of $\sigma$ with $\sigma$ gives us a torus knot 

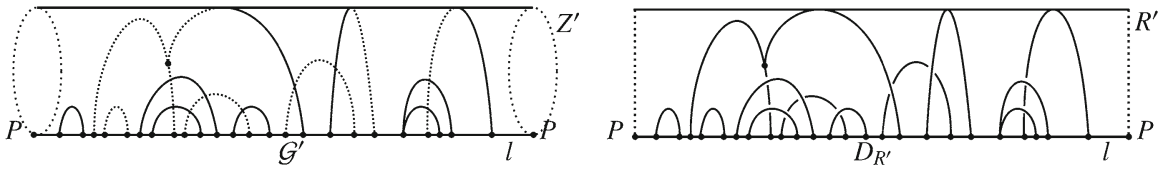

Fig. 6 The diagram $D_{R^{\prime}}$ of $\mathcal{G}^{\prime}$ obtained by projecting $Z^{\prime}$ onto $R^{\prime}$

$T^{\prime}(1, n)$. Since $T^{\prime}(1, n)$ is disjoint from $\pi_{\max }$, there exists a path $\pi_{c}$ consisting of $\pi$ and $T_{i}$ disjoint from $T^{\prime}(1, n)$ until some time after $t_{\text {max }}$. This contradicts the maximality of the pair we selected from $S$.

3. Deforming $T(1, n)$ to a longitude and finding a diagram $D_{R^{\prime}}$ of $\mathcal{G}$ :

Recall that the spatial graph $\mathcal{G}$ contains no nontrivial knots or links by assumption. By putting $\mathcal{G}$ into a general position, there exists a meridian $m$ of the torus that intersects $\mathcal{G}$ in exactly one point $P$. The existence of $P$ is given by the previous step (2). The point $P$ lies on $T(1, n)$ since every meridian intersects $T(1, n)$. Now perform the following twist: by cutting the torus $T$ along the meridian $m$, then twisting it $n$-times around the core of $T$ and identifying the same points again afterwards, an ambient isotopy $i: \mathcal{G} \rightarrow \mathcal{G}^{\prime}$ of the spatial graph is induced that maps $T(1, n)$ onto the longitude $l=T(1,0)$ of a new torus $T^{\prime}$ (not isotopic to $T$ ). We denote the image $i(\mathcal{G})$ on $T^{\prime}$ by $\mathcal{G}^{\prime}$. Restricted to the meridian $m$ of $T$, the isotopy is the identity by construction. Therefore, $\mathcal{G}^{\prime}$ and $m$ intersect in $l$ only. Define $Z^{\prime}:=T^{\prime} \backslash(m \backslash P)$.

Let us furthermore consider the diagram $D_{R^{\prime}}$ of $\mathcal{G}^{\prime}$ that we obtain the following way: We project $Z^{\prime}$ onto a rectangle $R^{\prime}=(0,1) \times[0,1] \cup\{(0,0)\} \cup\{(1,0)\}=$ $(l \backslash P) \times[0,1]) \cup\{P\}$ so that $l$ is the bottom line of $R^{\prime}$ and take a generic position so that the top line of $R^{\prime}$ does not intersect the graph in vertices. As usual, we indicate the over and under crossings of $\mathcal{G}^{\prime}$ (Fig. 6). Wlog we can assume that the diagram is regular, i.e. the diagram has only finitely many multiple points which all are transversal double points and no vertex is mapped onto a double point. Furthermore, let $D_{R^{\prime}}$ be a reduced diagram, i.e. a diagram with the minimal number of crossings that can be achieved from projecting $Z^{\prime}$ onto a rectangle $R^{\prime}$ as described.

4. Showing that pairs of spatial fragments in a reduced diagram $D_{R^{\prime}}$ have no crossings if they are non-conflicting and only one type of crossings if they are conflicting: By Tutte's Theorem 3, any cycle of an abstractly planar graph has a bipartite conflict graph. As $G$ is abstractly planar by assumption, it follows that the conflict graph of $l$ in $G$ is bipartite. As the graph $G$ is connected, all fragments of $G$ with respect to $l$ fall into two sets $S_{1}$ and $S_{2}$ so that fragments which are elements of the same set do not conflict. Choose an orientation of $l$. Starting at the point $P$, enumerate along the orientation all vertices $v_{1}, \ldots, v_{k}$ of $l$ that are endpoints of fragments of $G$ with respect to $l$. ( $P$ might or might not be the element of a fragment's endpointset.) Denote the spatial fragments of $\mathcal{G}^{\prime}$ by $f_{1}, \ldots, f_{n}$ respecting the orientation of $l$ and so that $v_{1} \in v\left(f_{1}\right)$. For each fragment $f_{i}$ of $G$ with respect to $l$, assign its endpointset $v\left(f_{i}\right)=v_{l_{i}} \leq \cdots \leq v_{r_{i}} \subset\left\{v_{1}, \ldots, v_{k}\right\}$. We show that each pair of 


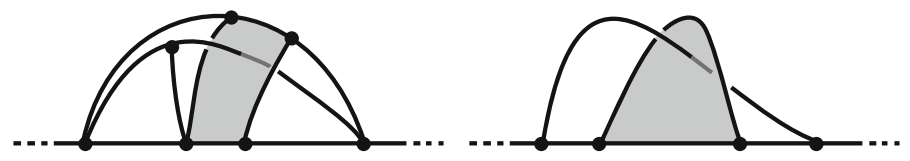

Fig. 7 A pair of conflicting spatial fragments in a reduced diagram $D_{R^{\prime}}$ with both over and under crossings does not embed on the torus

spatial fragments $f_{i}$ and $f_{j}$ has either no crossings or wlog $f_{i}$ over crosses $f_{j}$ at every crossing of the diagram $D_{R^{\prime}}$ :

(a) This is clear if $i=j$ since a fragment overcrosses (as well as undercrosses) itself at every self-crossing.

(b) If $i \neq j$ and $f_{i}$ and $f_{j}$ do not conflict, they have no crossings in a reduced diagram $D_{R^{\prime}}$ since one of the cases in Remark 1 holds: It is clear that there are no crossings between $f_{i}$ and $f_{j}$ in the first case of Remark 1. In the second case, let wlog $f_{j}$ be the inner fragment. Then, $f_{j}$ lies entirely inside the cell of $R^{\prime} \backslash f_{i}$ that has $\left[v_{l_{j}}, v_{r_{j}}\right]$ as part of the boundary. It follows from the connectivity of fragments that $f_{i}$ and $f_{j}$ have no crossings in a reduced diagram $D_{R^{\prime}}$.

(c) If $i \neq j$ and $f_{i}$ and $f_{j}$ are conflicting, only crossings of one type can occur. Since the entire spatial graph $\mathcal{G}^{\prime}$ is an embedding in $Z^{\prime}$ (as well as in $T^{\prime}$ ), it is not possible that both crossing types between $f_{i}$ and $f_{j}$ occur (Fig. 7). We remark that if $i \neq j$ and $f_{i}$ and $f_{j}$ are conflicting, they have at least one crossing in $D_{R^{\prime}}$ : Without affecting $D_{R^{\prime}}$, a fragment $\bar{f}$ can be added in $(0,1) \times[-1,0] \cup\{(0,0)\} \cup\{(1,0)\}$ so that $\bar{f}$ conflicts with both $f_{i}$ and $f_{j}$. Then, the subgraph consisting of $l, f_{i}, f_{j}$ and $\bar{f}$ is abstractly nonplanar by Tutte's Theorem 3 . The fragment $\bar{f}$ does not lie in $D_{R^{\prime}}$ by construction. Therefore, there exist neither crossings between $\bar{f}$ and $f_{i}$ nor between $\bar{f}$ and $f_{j}$. It follows from the abstract planarity that a crossing between $f_{i}$ and $f_{j}$ must exists.

5. Showing that a spatial fragment that conflicts with a pair of nested spatial fragments has the same crossing type with both of them:

If a fragment $f_{i}$ conflicts with two fragments $f_{j 1}$ and $f_{j 2}$, it follws from the bipartiteness of the conflict graph that $f_{j 1}$ and $f_{j 2}$ do not conflict. If $f_{j 1}$ and $f_{j 2}$ satisfy case (1) of Remark 1 , it cannot be concluded whether $f_{i}$ over or under crosses $f_{j 2}$ from knowing that $f_{i}$ over or under crosses $f_{j 1}$. However, if $f_{j 1}$ and $f_{j 2}$ are nested as in the second case of Remark 1 with $f_{j 1}$ being the inner fragment, and if $f_{i}$ wlog over crosses $f_{j 1}$ in $D_{R^{\prime}}$, then $f_{i}$ also over crosses $f_{j 2}$. We see this with a contradiction (Fig. 8): As in (c) of the step (4) above, there exists an element $v_{i a} \in v\left(f_{i}\right)$ so that $v_{l_{j 1}}<v_{a i}<v_{r_{j 1}}$. Assume that $f_{i}$ over crosses $f_{j 1}$ in a non-empty set of points but under crosses $f_{j 2}$ in a non-empty set in $D_{R^{\prime}}$. By the connectivity of fragments, there exists a path $p:[0,1] \rightarrow f_{i} \cup v_{i a} \cup v_{i b}$, with $v_{i b} \in v\left(f_{i}\right), a \neq b$ with endpoints $p(0)=v_{i a}$ and $p(1)=v_{i b}$, that over crosses $f_{j 1}$ in $p\left(t_{1}\right)$ and under crosses $f_{j 2}$ in $p\left(t_{2}\right)$. To change from an over to an under crossing, a path in $D_{R^{\prime}}$ has to intersect either the bottom or the top line of $R^{\prime}$. As $p$ does only intersect the bottom line in $v_{a i}$ and $v_{i b}, p$ must have an intersection 
Fig. 8 It is only possible to have different crossing types between a fragment and two nested fragments with whom the first fragment conflicts if the graph is not realized on the torus

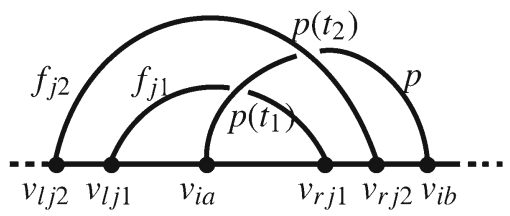

point $p(t)$ with the top line of $R^{\prime}$, so that $t_{1}<t<t_{2}$. But as $f_{j 1}$ is nested in $f_{j 2}$, it follows that $v_{l_{j 2}}<v_{i a}<v_{r_{j 2}}$. Therefore, $p$ starting from $v_{i a}$ overcrosses $f_{j 1}$ as well as undercrosses $f_{j 2}$ before it can intersect the top line of $R^{\prime}$. This is a contradiction.

6. Separating conflicting fragments to get a reduced diagram $D_{R^{\prime \prime}}$ in which no pair of fragments has crossings:

The conclusions made in (3) and (4) allow rotations of the spatial fragments of $\mathcal{G}^{\prime}$ around the longitude $l$ in $\mathbb{R}^{3}$ which gives an ambient isotopy from $\mathcal{G}^{\prime}$ to a realisation $\mathcal{G}^{\prime \prime}$ in which all fragments that are elements of $S_{1}$ lie on the torus $T^{\prime}$ and all elements of $S_{2}$ lie on a second torus $\hat{T}$. The torus $\hat{T}$ is glued to $T^{\prime}$ in $l$ along a longitude.

The rotations can be chosen as follows (compare Fig. 9): let $\left\{f^{1}\right\}$ be the set of all fragments of $\mathcal{G}^{\prime}$ with respect to $l$ (Fig. 9a) and order some variables as $1 \leq k_{11} \leq$ $\ldots \leq k_{t 1} \leq n$. If a step during the procedure which is decribed below cannot be performed, continue with the next step.

(a) Fix $f_{1}, \ldots, f_{k_{11}-1}$ where $f_{k_{11}}$ is the first fragment that conflicts with a fragment $f_{i}, 1 \leq i \leq k_{11}-1$ (Fig. 9a).

i. Set $\left\{f_{k_{11}, 1}\right\}=f_{k_{11}}$ and define $\left\{f_{k_{11}, r}\right\}, 2 \leq r \leq n$ as the union of $\left\{f_{k_{11}, r-1}\right\}$ and all fragments $f_{j}, k_{11} \leq j \leq n$ that are nested into $\left\{f_{k_{11}, r-1}\right\}$ or conflicting with $\left\{f_{k_{11}, r-1}\right\}$. Let $m_{1}$ be the smallest value of $r$ such that $\left\{f_{k_{11}, r}\right\}=\left\{f_{k_{11}, r+1}\right\}$.

Then rotate all spatial fragments that are elements of the set $\left\{f_{k_{11}, m_{1}}\right\}$ rigidly in $\mathbb{R}^{3}$ by $\pi$ around the longitude $l$. They are now embedded on $\hat{T}$. It is possible to choose the direction of the rotation so that no spatial fragments pass through each other since the spatial fragments in $\left\{f_{k_{11}, m_{1}}\right\}$ have only over crossings (respectively only under crossings) with $f_{1} \ldots f_{k_{11}-1}$ by construction and by steps (3) and (4) (Fig. 9c).

A. Let $f_{k_{12}}$ be the first fragment that is not an element of $\left\{f_{k_{11}, m_{1}}\right\}$ but conflicts with an element of $f_{1}, \ldots, f_{k_{11}-1}$. Define $\left\{f_{k_{12}, m_{2}}\right\}$ analogously to $\left\{f_{k_{11}, 1}\right\}$ (Fig. 9c). Since the elements of $\left\{f_{k_{11}, m_{1}}\right\}$ are neither nested nor conflicting with any elements of $\left\{f_{k_{12}, m_{2}}\right\}$, again, there is a rigid rotatation of $\left\{f_{k_{12}, m_{2}}\right\}$ in $\mathbb{R}^{3}$ by $\pi$ around the longitude $l$ that does not pass the spatial graph through itself (Fig. 9d).

B. Continue this procedure for all remaining spatial fragments that conflict with elements of $f_{1}, \ldots, f_{k_{11}-1}$ and denote the union of the sets $\left\{f_{k_{11}, m_{1}}\right\} \cup \ldots \cup\left\{f_{k_{1 s}, m_{s}}\right\}$ by $\left\{f^{k_{1}}\right\}$. Then, $\left\{f^{k_{1}}\right\}$ is embedded on $\hat{T}$ (Fig. 9d). 
(a)

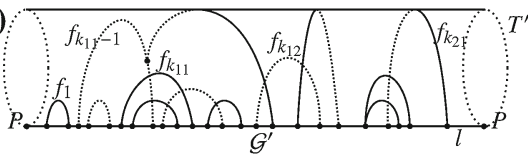

(c)

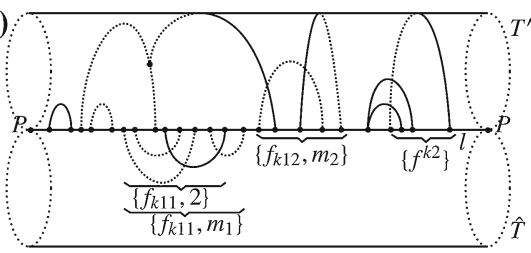

(e)

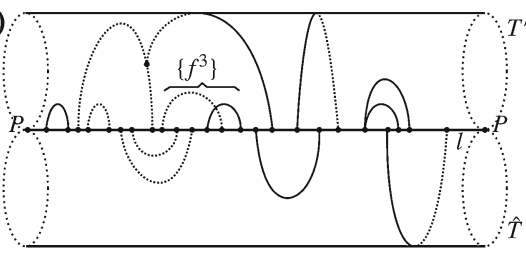

(b)

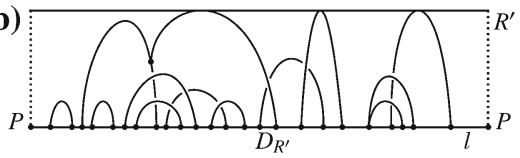

(d)

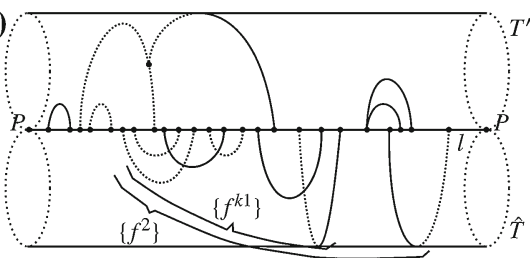

(f)

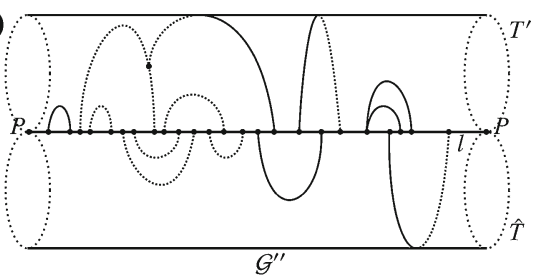

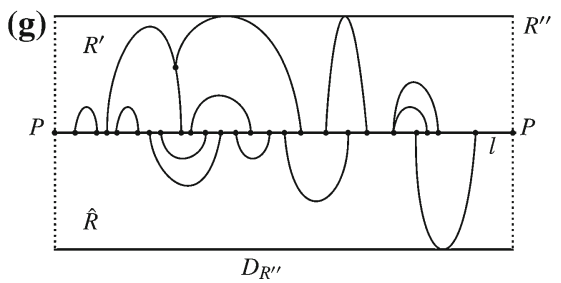

Fig. 9 The rotation of fragments described in (6). For clarity of the figure, each fragment is chosen not to have crossings with itself

ii. Let $f_{k_{21}} \notin\left\{f^{k_{1}}\right\}$ be the first fragment that conflicts with a fragment $f_{i}, i \leq k_{21}-1\left(f_{i} \notin\left\{f_{1}, \ldots, f_{k_{11}-1}\right\}\right.$ by construction). Fix $f_{1}, \ldots, f_{k_{21}-1}$. Define $\left\{f^{k_{2}}\right\}$ as above (Fig. 9c) and perform the rotation around $l$ (Fig. 9d).

iii. Continue with this procedure until all fragments $f_{1} \ldots f_{n}$ have been considered. The fragments that are elements of the set $\left\{f^{2}\right\}:=\left\{f^{k_{1}}\right\} \cup$ $\ldots \cup\left\{f^{k_{t}}\right\}$ are now embedded on $\hat{T}$ (Fig. 9d).

(b) Start (a) again beginning with the subgraph of $\mathcal{G}^{\prime}$ that corresponds to $\left\{f^{2}\right\}$. Note that during this step the rotations bring fragments back onto the torus $T^{\prime}$ but will not introduce crossings with $\mathcal{G}^{\prime}-\left\{f^{2}\right\}$ (Fig. 9e).

(c) Continue the procedure has to be continued until all elements of $S_{1}$ lie on the torus $T^{\prime}$ and all elements of $S_{2}$ lie on the torus $\hat{T}$ (Fig. 9f).

This gives a realisation $\mathcal{G}^{\prime \prime}$ of $G$ which is ambient isotopic to $\mathcal{G}$. By (3), a pair of spatial fragments of $\mathcal{G}^{\prime \prime}$ has no crossings in a reduced diagram $D_{R^{\prime \prime}}$ of $\mathcal{G}^{\prime \prime}$ on a rectangle $\left.\left.R^{\prime \prime}=(0,1) \times[-1,1]\right) \cup\{(0,0)\} \cup\{(1,0)\}=(l \backslash P) \times[-1,1] \cup\{P\}\right)$. The diagram $D_{R^{\prime \prime}}$ is the composition of two diagrams defined as in (2) for $T^{\prime}$ on $((l \backslash P) \times[0,1]) \cup\{P\})$ and analogously for $\hat{T}$ on $((l \backslash P) \times[-1,0]) \cup\{P\})$ (Fig. 9g). 

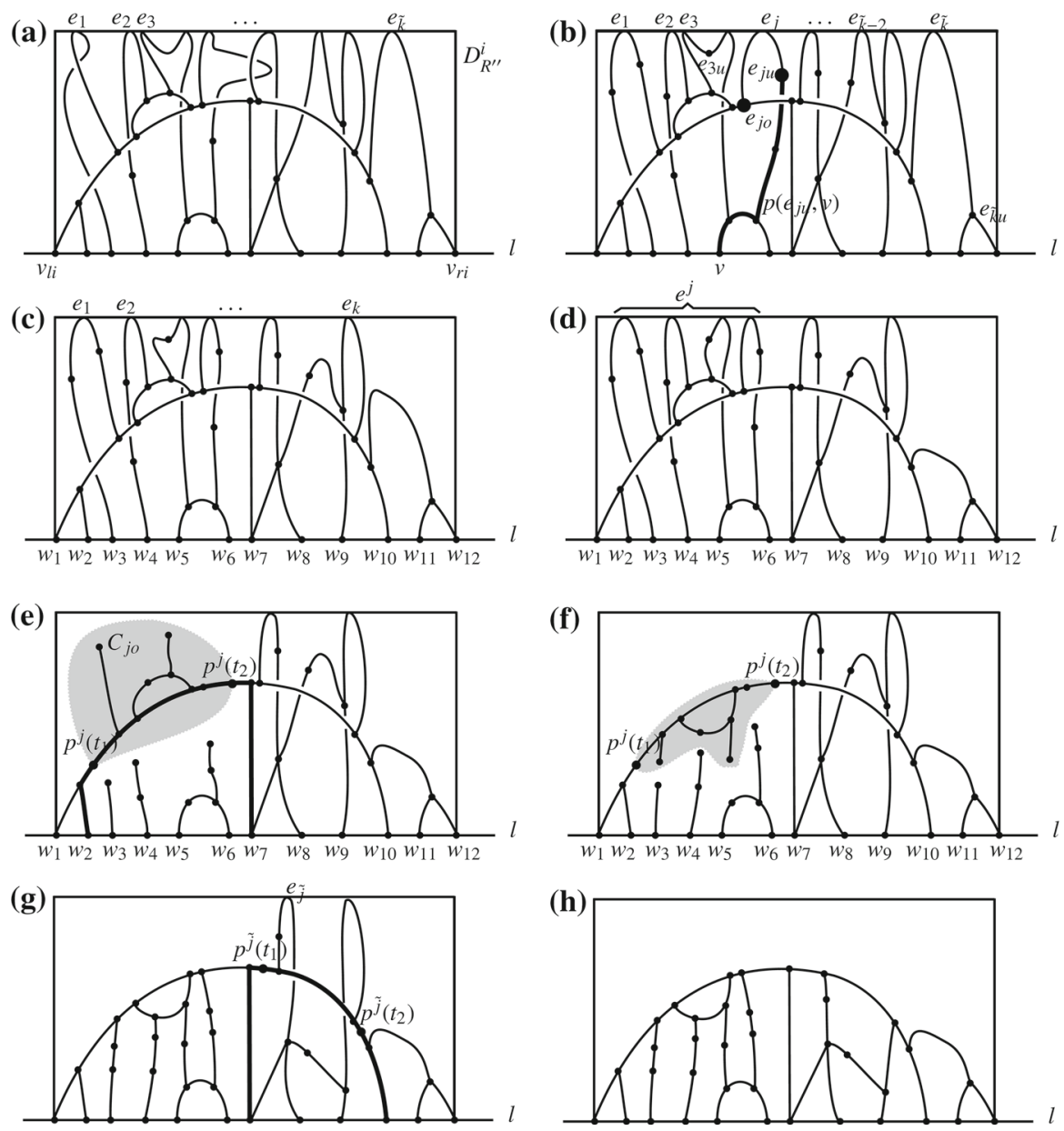

Fig. 10 Step 7: Deformation of the diagram $D_{R^{\prime \prime}}^{i}$ of a spatial fragment $f_{i}$ to a crossing free diagram

7. Showing that a single spatial fragment $f_{i}$ has no crossings in a reduced diagram $D_{R^{\prime \prime}}$ of $\mathcal{G}^{\prime \prime}$ (Fig. 10):

Each spatial subgraph $f_{i} \cup\left[v_{l i}, v_{r i}\right]$ is embedded on a sphere $S_{i}^{2}$. To see this, let wlog $f_{i}$ be embedded on $T^{\prime}$ and take two meridians of $T^{\prime}$ intersecting $l$ in $v_{l i}$ and $v_{r i}$ so that the meridians do not intersect $\mathcal{G}^{\prime \prime}$ except in $v_{l i}$ and $v_{r i}$. Then glue two meridional discs in, one in each meridian. $S_{i}^{2}$ consists of the two meridional discs and the part of $T^{\prime}$ where $f_{i}$ is embedded in that lies between the meridians. We now want to ambient isotope $f_{i}$ inside the ball bounded by $S_{i}^{2}$ where we take the inside to be the component of $\mathbb{R}^{3} \backslash S_{i}^{2}$ that does not intersect $\mathcal{G}^{\prime \prime}$. This isotopy will transform the diagram $D_{R^{\prime \prime}}$ to a diagram in which the subdiagram corresponding to $f_{i}$ that is crossing free.

Take the subdiagram $D_{R^{\prime \prime}}^{i}$ of the diagram $D_{R^{\prime \prime}}$ that corresponds to $f_{i} \cup\left[v_{l i}, v_{r i}\right]$. Peform all reducing Reidemeister I \& II moves on it (Fig. 10a, b). Simplify $D_{R^{\prime \prime}}^{i}$ 
by isotopy whenever possible. We can assume that the diagram $D_{R^{\prime \prime}}^{i}$ has edges crossing the top line of $R^{\prime \prime}$; otherwise it is crossing free and we are done. If one of those edges intersects the top line of $R^{\prime \prime}$ more than once or runs through a crossing in $D_{R^{\prime \prime}}^{i}$, we subdivide the edge by adding vertices (Fig. 10a, b). Therefore, we can assume that an edge of $D_{R^{\prime \prime}}^{i}$ that intersects the top line of $R^{\prime \prime}$ intersects it only once and is crossing free in the diagram. (This does not affect our argument since if a subdivision of a spatial graph is planar embedded, the spatial graph itself is.) Denote the edges crossing the top line of $R^{\prime \prime}$ by $\left\{e_{1}, \ldots, e_{\tilde{k}}\right\}$. Each edge $e_{j}$ has two endpoints, $e_{j u}$ and $e_{j o}$ (fat in Fig. 10b). By the connectivity of $\mathcal{G}^{\prime \prime}$, there is at least one edge $e_{j}$ in $\left\{e_{1}, \ldots, e_{\tilde{k}}\right\}$ for which there exists a path in $f_{i}$ from an endpoint of the edge wlog $e_{j u}$ to an element $v \in v\left(f_{i}\right)$ that does not intersect the top line of $R^{\prime \prime}$. Denote such a path with endpoints $e_{j u}$ and $v$ by $p\left(e_{j u}, v\right)$ (fat in Fig. 10b). The set of all such paths is called $\{p(j u)\}$. The set $\{p(j o)\}$ is analogously defined for the endpoint $e_{j o}$ of $e_{j}$.

If an edge $e_{j} \in\left\{e_{1}, \ldots, e_{\tilde{k}}\right\}$ has an endpoint $e_{j u}$ or $e_{j o}$ which is not the endpoint of any path in $\{p(j u)\}$ or $\{p(j o)\}\left(e_{3 u}\right.$ in Fig. 10b), $e_{j}$ can be deformed not to intersect the top line of $R^{\prime \prime}$ by moving $e_{j}$ away from the top line while keeping $D_{R^{\prime \prime}}-e_{j}$ fixed. After this procedure, a subset of edges $\left\{e_{1}, \ldots, e_{k}\right\} \subseteq\left\{e_{1}, \ldots, e_{\tilde{k}}\right\}$ remains in which every edge $e_{j}$ has endpoints $e_{j u}$ and $e_{j o}$ so that there exists at least two (possibly constant) paths $p\left(e_{j u}, v_{a}\right)$ and $p\left(e_{j o}, v_{b}\right)$ with $v_{a}, v_{b} \in v\left(f_{i}\right)$. Such a path $p\left(e_{j u}, v_{a}\right)$ or $p\left(e_{j o}, v_{b}\right)$ cannot have both over crossings and under crossings since in this case the path would intersect $\partial R^{\prime \prime}$ which it does not by construction. Also by construction, if a path in $\{p(j x)\}, x=u, o$ has an over crossing (or respectively under crossing), no path in $\{p(j x)\}$ has an under (respectively over) crossing. In addition, we can assume wlog that there is no edge $e_{j} \in\left\{e_{1}, \ldots, e_{k}\right\}$ that has an endpoint $e_{j u}$ or $e_{j o}$ so that all paths $\{p(j u)\}$ or $\{p(j o)\}$ are crossing free $\left(e_{\tilde{k} u}\right.$ in Fig. 10b) as in this case we can deform $e_{j}$ away from the top line while keeping $D_{R^{\prime \prime}}-e_{j}$ fixed. Also, if $\{p(j u)\}$ and $\{p(j o)\}$ have only one type of crossings ( $e_{\tilde{k}-2}$ in Fig. 10b), we can again deform $e_{j}$ away from the top line while keeping $D_{R^{\prime \prime}}-e_{j}$ fixed. Therefore, every edge $e_{j}$ has one endpoint $e_{j u}$ so that at least one path in $\{p(j u)\}$ has crossings which all are under crossings and one endpoint $e_{j o}$ so that at least one path in $\{p(j o)\}$ has crossings which all are over crossings. Furthermore, in $\{p(j u)\}$ (respectively $\{p(j o)\}$ ) are no paths that have over crossings (respectively under crossings) by definition of the paths.

Assign to each edge $e_{j} \in\left\{e_{1}, \ldots, e_{k}\right\}$ the set $w\left(e_{j u}\right) \subseteq\left\{w_{j 1}, \ldots, w_{j l}\right\}$ (analogously $\left.w\left(e_{j o}\right) \subseteq\left\{w_{j 1}, \ldots, w_{j l}\right\}\right)$ which is the set of points in $v\left(f_{i}\right)$ that are endpoints of at least one element in $\{p(j u)\}$ (respectively $\{p(j o)\}$ ). The union of the two sets is the endpointset of $e_{j}$ denoted by $w\left(e_{j}\right)=\left\{w_{j 1}, \ldots, w_{j l}\right\}:=$ $w\left(e_{j u}\right) \cup w\left(e_{j o}\right)$. The sets in the example in Fig. 10c are $w\left(e_{k u}\right)=\left\{w_{7}, w_{8}, w_{9}\right\}$ and $w\left(e_{k o}\right)=\left\{w_{1}, w_{2}, w_{7}, w_{10}, w_{11}, w_{12}\right\}$. Denote the union of $w\left(e_{1 u}\right) \cup \ldots \cup$ $w\left(e_{k u}\right)$ by $w(u)$ (and the union $w\left(e_{1 o}\right) \cup \ldots \cup w\left(e_{k o}\right)$ by $\left.w(o)\right)$. (In Fig. 10c), $\left.w(u)=\left\{w_{3}, w_{4}, w_{5}, w_{6}, w_{7}, w_{8}, w_{9}\right\}, w(o)=\left\{w_{1}, w_{2}, w_{7}, w_{10}, w_{11}, w_{12}\right\}\right)$

See that there exist no four points $w_{u 1}, w_{u 2} \in w\left(e_{j u}\right)$ and $w_{o 1}, w_{o 2} \in w\left(e_{j o}\right)$ that are interlaced as wlog $w_{u 1}<w_{o 1}<w_{u 2}<w_{o 2}$ : the cycle $\left(l-\left(w_{u 1}, w_{o 2}\right)\right.$, $\left.p\left(e_{j u}, w_{u 1}\right), e_{j}, p\left(e_{j o}, w_{o 1}\right),\left[w_{o 1}, w_{u 2}\right],\left[w_{u 2}, w_{o 2}\right]\right)$ would have three pairwise 
Fig. 11 The situation where points in which $\{p(j u)\}$ and $\{p(j o)\}$ are attached to the circle $l$ are interlaced as drawn in the figure cannot occur

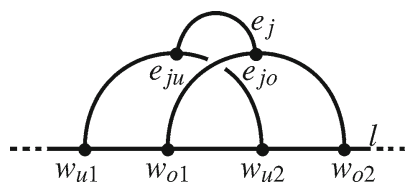

conflicting fragments $\left[w_{u 1}, w_{o 1}\right], p\left(e_{j u}, w_{u 2}\right)$ and $p\left(e_{j o}, w_{o 2}\right)$ (Fig. 11). This contradicts the bipartiteness of $\mathcal{G}^{\prime \prime}$ which by Theorem 3 contradicts its abstract planarity. (Also, this graph forms $K_{3,3}$ where the points $w_{u 1}, e_{j o}, w_{u 2}, w_{o 1}, e_{j u}, w_{o 2}$ are the vertices.) Therefore, $w\left(e_{j u}\right)$ and $w\left(e_{j o}\right)$ can only be arranged like the endpointsets in Remark 1 and it is allowed to restrict to those cases as done below. By the connectivity of fragments, each element of $v\left(f_{i}\right)$ belongs to $w(u)$, $w(o)$ or to both. This gives a division of $\left[v_{l i}, v_{r i}\right]$ into intervals, where a new interval starts at each point of $v\left(f_{i}\right)$ that is an element of both $w(u)$ and $w(o)$ or where a new interval starts in a point $v_{k+1}$ if $w(o) \not \supset v_{k} \in w(u)$ and $w(u) \not \supset v_{k+1} \in w(o)$ (or if $u$ and $o$ exchanged). (In Fig. 10d, the intervals are $\left.\left[w_{1}, w_{3}\right],\left[w_{3}, w_{7}\right],\left[w_{7}, w_{10}\right],\left[w_{10}, w_{12}\right].\right)$

The setting is now sufficiently well understood to eliminate all remaining crossings in two cases:

Case 1: $w\left(e_{j u}\right)$ and $w\left(e_{j o}\right)$ are nested.

Assume that for an edge $e_{j}, w\left(e_{j u}\right)$ and $w\left(e_{j o}\right)$ are nested with wlog $w\left(e_{j u}\right)$ being the inner points. The inner points are all contained in one interval of the bottom line division since if they laid in two intervals, there would exist a point $w\left(e_{j o}\right) \not \supset$ $w_{m} \in w(o)$ between two points of $w\left(e_{j u}\right)$. Consequently, there would exist a path $p\left(e_{j^{\prime} o}, w_{m}\right), j \neq j^{\prime}$ with endpoints $e_{j^{\prime} o}$ and $w_{m}$ which does not intersect any of the paths that are elements of $w\left(e_{j o}\right)$. This is not possible as $R^{\prime \prime} \backslash\left(p\left(e_{j^{\prime} o}, w_{m}\right) \cup e_{j^{\prime}}\right)$ consists of two components of which both contain elements of $w\left(e_{j u}\right)$ and there exists a paths between any point of $w\left(e_{j u}\right)$ and $e_{j u}$ by definition.

Define $\left\{e^{j}\right\}$ as the subset of edges $\left\{e^{j}\right\} \subseteq\left\{e_{1}, \ldots, e_{k}\right\}$ so that all edges in $\left\{e^{j}\right\}$ have an endpoint in the interval $I$ of the bottom line that contains points of $w\left(e_{j u}\right)$ (Fig. 10d: $e^{1}=e^{2}=e^{3}=e^{4} \neq e^{5}=e^{6}$ ). Now consider the diagram $D_{R^{\prime \prime}}-\left\{e^{j}\right\}$ in which all edges that are elements of the set $\left\{e^{j}\right\}$ are deleted (Fig. 10e). There exists a path $p^{j}:[0,1] \rightarrow D_{R^{\prime \prime}}-\left\{e^{j}\right\}$ from $p^{j}(0) \in w\left(e_{j o}\right)$ to $p(1) \in w\left(e_{j o}\right)$ such that $p^{j}(0) \leq w\left(e_{j u}\right) \leq p^{j}(1)$ and so that there exist two distinct points $p^{j}\left(t_{1}\right), p^{j}\left(t_{2}\right)$ with $t_{1}, t_{2} \in[0,1]$ that have the following property: The diagram $D_{R^{\prime \prime}}-\left\{e^{j}\right\}-$ $p^{j}\left(t_{1}\right)-p^{j}\left(t_{2}\right)$ splits such that the component $C_{j o}$ containing $e_{j o}$ does not contain any points of $\left[v_{l i}, v_{r i}\right]$ (Fig. 10e). Furthermore, after performing a Whitney 2-flip on $C_{j o}$ (Fig. 10e, f), the edges of $\left\{e^{j}\right\}$ can be reintroduced to the diagram $D_{R^{\prime \prime}}-\left\{e^{j}\right\}$ without introducing crossings (Fig. 10g). A Whitney 2-flip replaces a component by its mirror image as shown in Fig. 12, left. This corresponds to a rotation in $\mathbb{R}^{3}$ by $\pi$ that would not pass the spatial graph $\mathcal{G}^{\prime \prime}$ through itself - even if all edges $\left\{e^{j}\right\}$ are left attached (Fig. 12, right and Fig. 10d-g). Therefore, we have an ambient isotopy, that eliminates the crossings of $\left\{e^{j}\right\}$.

After continuing this procedure, all remaining edges of $\left\{e_{1}, \ldots, e_{k}\right\}$ have endpointsets so that for each edge all elements of $w\left(e_{j u}\right)$ are smaller or equal than all 

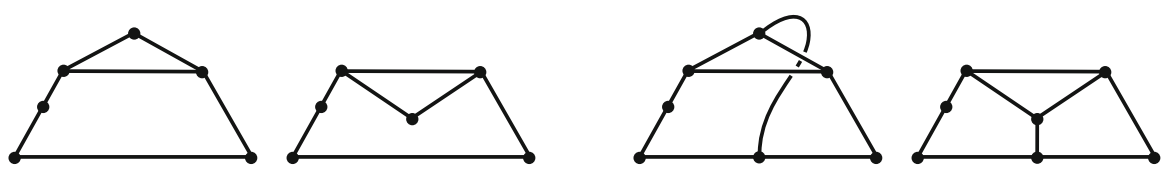

Fig. 12 Whitney 2-flip and corresponding rotation by $\pi$

Fig. 13 Contradiction showing that $p\left(t_{1}\right)$ and $p\left(t_{2}\right)$ do exist

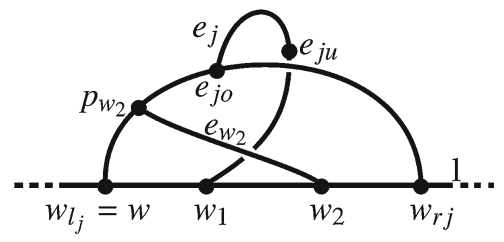

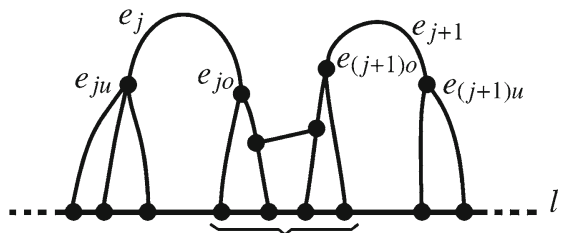

$w\left(e_{j o}\right)=w\left(e_{(j+1) o}\right)$

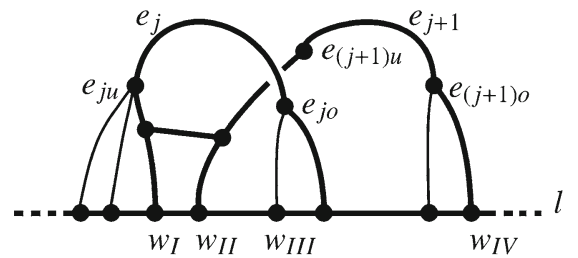

Fig. 14 Case 2: $w\left(e_{j u}\right) \leq w\left(e_{j o}\right)$. The left figure is planar. The right situation cannot occur since the graph is not abstractly planar

elements of $w\left(e_{j o}\right)$ (or all elements of $w\left(e_{j u}\right)$ are greater or equal than all elements of $\left.w\left(e_{j o}\right)\right)$.

See with a contradiciton that it is always possible to find two points $p\left(t_{1}\right)$ and $p\left(t_{2}\right)$ with the required property as follows. If no two points $p\left(t_{1}\right)$ and $p\left(t_{2}\right)$ with the required property would exist, there existed a subgraph of $\mathcal{G}^{\prime \prime}$ that is abstractly nonplanar (Fig. 13): Wlog there would exist two points $w\left(e_{j u}\right) \ni w_{1}<w_{2} \in$ $w\left(e_{j o}\right)$ such that there would exist an edge $e_{w 2}$ between $w_{2}$ and an inner point $p_{w 2}$ of a path $\stackrel{\circ}{p}\left(e_{j o}, w\right)$ with $w<w_{1}$. Let $0<t_{s}<1$ such that $p\left(e_{j o}, w\right)\left(t_{s}\right)=p_{w 2}$. This gives a cycle with non-bipartite conflict graph (alternatively, see $K_{3,3}$ ): The cycle consists of the following segments: $l-\left(w, w_{r j}\right),\left.p\right|_{[0, s]}, e_{w 2},\left[w_{1}, w_{2}\right]$, $p\left(w_{1}, e_{j u}\right), e_{j}, p\left(e_{j o}, w_{r j}\right)$ and the fragments are $\left(w, w_{1}\right),\left.p\right|_{(s, 1)}$ and $\left(w_{2}, w_{r j}\right)$. This contradicts abstract planarity by Theorem 3 .

Case 2: All endpoints in $w\left(e_{j u}\right)$ are smaller or equal to all endpoints in $w\left(e_{j o}\right)$ (or respectively $\left.w\left(e_{j u}\right) \geq w\left(e_{j o}\right)\right)$.

If $\left\{e_{1}, \ldots, e_{k}\right\}$ is empty or has one element only, it follows that the diagram of $f_{i}$ has no crossings. So consider the case that $k \geq 2$. Wlog, assume that all elements of $w\left(e_{j u}\right)$ are smaller or equal to all elements of $w\left(e_{j o}\right)$. If all elements of $w\left(e_{(j+1) u}\right)$ are greater or equal to all elements of $w\left(e_{(j+1) o}\right)$, it follows that $w\left(e_{j o}\right)=w\left(e_{(j+1) o}\right)$ by construction and the connectivity of fragments (Fig. 14, left). If all elements of $w\left(e_{(j+1) u}\right)$ are smaller or equal to all elements of $w\left(e_{(j+1) o}\right)$ but there exists an element $w_{I I} \in w\left(e_{(j+1) u}\right)$ that is smaller than an element $w_{I I I} \in w\left(e_{j o}\right)$, there are four elements $w_{I}<w_{I I}<w_{I I I}<$ $w_{I V}, w_{I} \in w\left(e_{j u}\right), w_{I V} \in w\left(e_{(j+1) o}\right)$. The paths $p\left(w_{I}, e_{j u}\right), e_{j}, p\left(w_{j o}, e_{I I I}\right)$ 
and $p\left(w_{I I}, e_{(j+1) u}\right), e_{j+1}, p\left(w_{j o}, e_{I V}\right)$ are connected via a path in $\stackrel{R}{\prime}^{\prime \prime}$ by the connectivity of fragments. The subgraph of $\mathcal{G}^{\prime \prime}$ (fat in Fig. 14, right) consisting of those three paths and $l$ is abstractly nonplanar (it is $K_{3,3}$ ) which can again be shown with a similar argument as above by choosing any Hamilton cycle of the subgraph (i.e. a cycle that runs through every vertex of the subgraph once) and seeing that its conflict graph is not bipartite.

Therefore, it is shown in step (7) that a reduced form of the diagram $D_{R}^{\prime \prime}$ has no crossings. It follows together with step (6) that $\mathcal{G}^{\prime \prime}$ and therefore $\mathcal{G}^{\prime}$ is planar embedded. The argument of step (6) relies on step (5) and step (4). By step (3), which can be performed because of step (2), $\mathcal{G}$ is also planar embedded. This proves the theorem by step (1).

Corollary 2 (Ravels do not embed on the torus) Every nontrivial embedding of $\theta_{n^{-}}$ graphs on the torus contains a nontrivial knot.

Proof As there exist no pair of disjoint cycles in a $\theta_{n}$-graph, such a graph does not contain a nonsplit link. Since $\theta_{n}$-graphs are planar, the statement of the corollary follows directly from Theorem 1 .

\subsection{Alternative proof of Theorem 1}

The proof of Theorem 1 given above can be differently finished using Theorem 4 by $\mathrm{Wu}[30]$. This is a shortcut in the argument but does not give an explicit deformation.

Theorem 4 (Criterion for planar embeddedness of an anbstractly planar graph [30]) An abstractly planar graph is planar embedded if and only if every cycle in the spatial graph bounds an embedded disc with interior disjoint from the spatial graph.

\section{Alternative proof of Theorem 1.}

Proof Start with step (1) and step (2) of the proof that is given in the section above. Recall that the point $P$ is defined as follows: beside elementary cases, there exists a meridian of the torus on which the spatial graph $\mathcal{G}$ is embedded on so that the meridian intersects the spatial graph in only one point. Take this point to be $P$. To apply Theorem 4 , observe that every cycle in $\mathcal{G}$ bounds a disc embedded in $\mathbb{R}^{3}$ with interior disjoint from $\mathcal{G}$. This is clearly true for any meridian and for any cycle that does not intersect $P$ as $\mathcal{G} \backslash P$ is embedded on a sphere already. We are left to consider cycles $C$ that run through $P$ for which there exists a natural number $n$ so that the cycle has knot type $T(n, 1)$. Let $C_{n}$ be one of those cycles. We can find an ambient isotopy $i_{n}$ of $\mathcal{G}$ that transforms $C_{n}$ to a longitude $l=i_{n}\left(C_{n}\right)$ of a new torus $i_{n}(T)$ (not isotopic to $T$ ), by possibly performing another twist as described in step (3) of the proof above. Denote the spatial graph that results from this twist by $i_{n}(\mathcal{G})$. As a longitude bounds a disc internally disjoint from the torus, it follows that the cycle $i_{n}\left(C_{n}\right)$ bounds a disc internally disjoint from $i_{n}(\mathcal{G})$. Since ambient isotopies preserve embedded discs and do not pass them through the graph, it follows that the cycle $C_{n}$ in $\mathcal{G}$ bounds a disc internally disjoint from $\mathcal{G}$. For every $n \in \mathbb{N}$, we can perform such an ambient isotopy of $\mathcal{G}$. This shows that every cycle in $\mathcal{G}$ bounds a disc which is internally disjoint from 
the spatial graph. As $G$ is abstractly planar by assumption, it follows from Theorem 4 that $\mathcal{G}$ is planar embedded.

Remark 2 It is not possible to weaken the assumptions of Theorem 1 as shown by giving counter examples in [25].

Acknowledgments The first author thanks Tom Coates, Erica Flapan, Youngsik Huh, Stephen Hyde, Danielle O'Donnol, Makoto Ozawa, and Kouki Taniyama for helpful comments and discussions and is very grateful for the extensive comments of the first reviewer that improved the quality of the paper a lot. Matt Rathbun is particularly thanked for long discussions and the outline of the second step in the proof. The research was financially supported by the Roth studentship of Imperial College London mathematics department, the DAAD, the Evangelisches Studienwerk, the Doris Chen award, and by a JSPS grant awarded to Kouki Taniyama.

Open Access This article is distributed under the terms of the Creative Commons Attribution 4.0 International License (http://creativecommons.org/licenses/by/4.0/), which permits unrestricted use, distribution, and reproduction in any medium, provided you give appropriate credit to the original author(s) and the source, provide a link to the Creative Commons license, and indicate if changes were made.

\section{References}

1. J.-P. Sauvage, D.B. Amabilino, Templated synthesis of knots and ravels, in Supramolecular Chemistry: From Molecules to Nanomaterials, vol. 5, ed. by P. Gale, J. Steed (Wiley, Hoboken, 2012), p. 2245

2. R.S. Forgan, J.-P. Sauvage, J.F. Stoddart, Chemical topology: complex molecular knots, links, and entanglements. Chem. Rev. 111, 54345464 (2011)

3. D.B. Amabilino, L. Pérez-García, Topology in molecules inspired, seen and represented Chem. Soc. Rev. 38, 1562-1571 (2009)

4. E.E. Fenlon, Open problems in chemical topology. Eur. J. Org. Chem. 30, 5023-5035 (2008)

5. D. Andrae, Molecular knots, links, and fabrics: prediction of existence and suggestion of a synthetic route. New J. Chem. 30, 873-882 (2006)

6. L. Carlucci, G. Ciani, D.M. Proserpio, Polycatenation, polythreading and polyknotting in coordination network chemistry. Coord. Chem. Rev. 246, 247-289 (2003)

7. E. Flapan, When Topology Meets Chemistry (Cambridge University Press, Cambridge, 2000)

8. J.-F. Ayme, J.E. Beves, D.A. Leigh, R.T. McBurne, K. Rissanen, D. Schultz, Pentameric circular iron(II) double helicates and a molecular pentafoil knot. J. Am. Chem. Soc. 22, 9488-9497 (2012)

9. F. Li, J.C. Clegg, L.F. Lindoy, R.B. Macquart, G.V. Meehan, Metallosupramolecular self-assembly of a universal 3-ravel. Nat. Commun. 2, 205.1-205.5 (2011)

10. C.D. Pentecost, K.S. Chichak, A.J. Peters, G.W.V. Cave, S.J. Cantrill, J.F. Stoddart, A molecular solomon link. Angew. Chem. Int. Ed. 46, 218-222 (2007)

11. M. Feigel, R. Ladberg, S. Engels, R. Herbst-Irmer, R. Fröhlich, A trefoil knot made of amino acids and steroids. Angew. Chem. Int. Ed. Engl. 45, 5698-5702 (2006)

12. J.S. Siegel, Chemical topology and interlocking molecules. Science 304, 1256-1258 (2004)

13. R.F. Carina, C. Dietrich-Buchecker, J.-P. Sauvage, Molecular composite knots. J. Am. Chem. Soc. 118, 9110-9116 (1996)

14. J.F. Nierengarten, C.O. Dietrich-Buchecker, J.P. Sauvage, Copper(I) template synthesis of a 4-crossing 2-catenane. New J. Chem. 44, 1456-1477 (1996)

15. S. Du, N. Seeman, Synthesis of a DNA knot containing both positive and negative nodes. J. Am. Chem. Soc. 114, 9652-9655 (1992)

16. C.O. Dietrich-Buchecker, J.-P. Sauvage, A synthetic molecular trefoil knot. Angew. Chem. Int. Ed. Engl. 28, 189-192 (1989)

17. D.M. Walba, R.M. Richards, R.C. Haltiwanger, Total synthesis of the first molecular Moebius strip. Math. J. Am. Chem. Soc 104, 32193221 (1982)

18. L.A. Paquette, M. Vazeux, Threefold transannular epoxide cyclization. Synthesis of a heterocyclic $C_{17}$-hexaquinane. Tetrahedron Lett. 22, 291-294 (1981) 
19. H.E. Simmons III, J.E. Maggio, Synthesis of the first topologically non-planar molecule. Tetrahedron Lett. 22, 287-290 (1981)

20. V.A. Blatov, L. Carlucci, G. Ciani, D.M. Proserpio, Interpenetrating metalorganic and inorganic 3D networks: a computer-aided systematic investigation. Part I. Analysis of the Cambridge structural database. Cryst. Eng. Comm. 6, 377-395 (2004)

21. I.A. Baburin, V.A. Blatov, L. Carlucci, G. Ciani, D.M. Proserpio, Interpenetrating metal-organic and inorganic 3D networks: a computer-aided systematic investigation. Part II. Analysis of the inorganic crystal structure database (ICSD). J. Solid State Chem. 178, 2452-2474 (2005)

22. T. Castle, M.E. Evans, S.T. Hyde, Ravels: knot-free but not free. Novel entanglements of graphs in 3-space. New J. Chem. 32, 1484-1492 (2008)

23. C. Farkas, E. Flapan, W. Sullivan, Unravelling tangled graphs. J. Knot Theory Ramif. 21, 1250074 (2012)

24. T. Castle, M.E. Evans, S.T. Hyde, All toroidal embeddings of polyhedral graphs in 3-space are chiral. New J. Chem. 33, 2107-2113 (2009)

25. S. Barthel, There exist no minimally knotted planar spatial graphs on the torus. J. Knot Theory Ramif. (2014). doi:10.1142/S0218216515500352

26. S. Kinoshita, On elementary ideals of polyhedra in the 3-sphere. Pac. J. Math. 42, 89-98 (1972)

27. S. Suzuki, Almost unknotted $\theta_{n}$-curves in the 3-sphere. Kobe J. Math. 1, 19-22 (1984)

28. S. Barthel, On chirality of toroidal embeddings of polyhedral graphs in 3-space (2014, preprint). arXiv: 1505.06040

29. W.T. Tutte, A homotopy theorem for matroids. Trans. Amer. Math. Soc. 88, 144160-161174 (1958)

30. Y. Wu, On planarity of graphs in 3-manifolds. Comment. Math. Helv. 67, 635-647 (1992) 